\title{
Serratia Marcescens Infection Complicating Peritoneal Dialysis
}

\author{
A. W. MCCRACKEN,* M.B., CH.B., D.C.P. ; F. E. LIPSCOMB,† C.B., C.B.E., M.R.c.s., L.R.C.P., D.T.M.\&H., D.P.H.
}

Brit. med. F., 1965, 1, 1536-1537

Serratia marcescens (Chromobacterium prodigiosum) is a freeliving saprophyte generally regarded as being non-pathogenic (Wilson and Miles, 1955). There is, however, reason to believe that under certain conditions this organism may be pathogenic.

Three instances are here reported in which $S$. marcescens infection of the peritoneum occurred during peritoneal dialysis for acute renal failure. All three patients were transferred to Princess Mary's Royal Air Force Hospital, Halton, for management of their renal failure.

\section{Case 1}

A man aged 59 was admitted to hospital on account of progressive oliguria and back pain of one week's duration. On admission he was drowsy, oedematous, and slightly cyanosed. His urinary output during the first 24 hours was only $70 \mathrm{ml}$., but the urine was sterile on culture. Blood urea was $240 \mathrm{mg} . / 100 \mathrm{ml}$. and serum potassium had reached $8.9 \mathrm{mEq} / \mathrm{l}$. Electrocardiogram showed gross hyperkalaemic changes. A 34-hour peritoneal dialysis was carried out, during which the urea and potassium levels fell to $190 \mathrm{mg} . / 100 \mathrm{ml}$. and $7.1 \mathrm{mEq} / 1$. respectively, but with little improvement in the clinical condition.

The first sample of dialysate was sterile, but samples taken at a later period in the dialysis grew Escherichia coli sensitive to polymyxin E (colistin), with which treatment was begun. Four days later a six-hour haemodialysis with a Kolff artificial kidney was carried out, again with a satisfactory fall in serum potassium and urea levels. Despite this the patient remained in a semicomatose state and a further four days later another peritoneal dialysis was carried out. On this occasion turbid fluid was obtained from the peritoneal catheter. From this fluid $E$. coli and $S$. marcescens were cultured on initial sampling, and two days later a pure culture of the latter organism was obtained.

The patient now had slight abdominal distension and infrequent bowel sounds. He remained anuric, and, despite continuance of antibiotic therapy and a further haemodialysis, he died 21 days after admission.

At necropsy the peritoneal cavity contained $1,200 \mathrm{ml}$. of turbid fluid and there were numerous recent adhesions between coils of small bowel. Culture of the fluid and a swab of the peritoneal surface gave a pure growth of $S$. marcescens. The kidneys were normal in size but showed considerable cortical scarring. Histologically, the peritoneum in a variety of sites showed acute inflammatory changes with substantial amounts of fibrinous exudate, while both kidneys showed the changes of acute-on-chronic pyelonephritis.

\section{Case 2}

A 22-year-old housewife was admitted with a history of abdominal discomfort, haematuria, and a rash on her face and hands, all of which had been present for several weeks. Shortly before admission she had several fits and periods of drowsiness associated with progressive oliguria and rising blood urea and serum potassium levels. Peritoneal dialysis was carried out four days after her admission. No difficulty was encountered until the second day of the dialysis, when aspiration of fluid through the abdominal catheter became difficult. Samples of the dialysate, initially sterile, now yielded a mixed growth of $S$. marcescens and $E$. coli, the latter sensitive to polymyxin E. There were now signs of early peritonitis

* Squadron Leader, R.A.F.; Royal Air Force Institute of Pathology and Tropical Medicine, Halton, Aylesbury, Bucks.

t Air Vice-Marshal (ret.), R.A.F.; Royal Air Force Institute of Pathology and Tropical Medicine, Halton, Aylesbury, Bucks. and treatment with polymyxin $\mathrm{E}$ was begun. A haemodialysis was carried out three days after peritoneal dialysis. The patient's renal function began to improve, and this was associated with resolution of the peritonitis. Meanwhile renal biopsy had established a diagnosis of periarteritis nodosa. Her subsequent course was satisfactory, and on recent review was symptom-free with fairly good renal function.

\section{Case 3}

A 27-year-old clerk was admitted with symptoms of uraemia and with marked oliguria. Conservative treatment with Resonium and rigid fluid balance control had not resulted in improvement, so peritoneal dialysis was carried out on the day after his admission. During initial catheterization of the abdomen the presence of fluid in the peritoneum was noted. A pure growth of Pseudomonas aeruginosa sensitive to polymyxin $\mathrm{E}$ was obtained from this fluid. Treatment with polymyxin $\mathrm{E}$ was begun, but, although the culture of fluid taken the following day was sterile, all later cultures yielded a mixed growth of $S$. marcescens and Ps. aeruginosa. From the time of admission the patient's condition grew steadily worse and, despite some improvement following dialysis, he died four days after admission.

At necropsy numerous fresh peritoneal adhesions with a small amount of fluid were found in the abdomen. A mixed growth of S. marcescens and Ps. aeruginosa was obtained from the peritoneum. Macroscopically the kidneys were pale and swollen. Microscopically the peritoneum showed acute inflammatory changes, while both kidneys showed acute necrotizing glomerulonephritis.

\section{Bacteriological Findings}

In all three cases $S$. marcescens grew equally well aerobically at $22^{\circ}$ and $37^{\circ} \mathrm{C}$. but produced its characteristic red pigment only at $22^{\circ} \mathrm{C}$. The organisms were motile and Gram-negative, fermenting glucose, maltose, and salicin without gas production. Lactose was not fermented and indole was not produced. The organism isolated from each of the three patients was resistant to polymyxin $\mathrm{E}$ but sensitive to neomycin and kanamycin. The strain from Case 3 was, in addition, sensitive to ampicillin and chloramphenicol. Since Cases 1 and 2 were under treatment at the same time, it seems likely that both were infected by the same strain. Case 3, however, was admitted some five months later, and this, combined with the different sensitivity pattern, suggests infection by another strain.

Search for a source of the organism in the fluids used for dialysis, in the apparatus, and in the environment proved fruitless.

\section{Discussion}

There is a considerable amount of evidence that $S$. marcescens is essentially non-pathogenic. Klein (1894) reported gross contamination of food by this organism in a London mercantile establishment without ill-effects occurring in the large number of persons who ate the contaminated food. Both Burket and Burn (1937) and McEntegart and Porterfield (1949) were sufficiently sure of its lack of virulence to use the organism experimentally to demonstrate bacteriaemia after dental extraction. There were no reported ill-effects in their volunteers. Against this, Paine (1946) described mild respiratory illness following inhalation of $S$. marcescens by volunteers. This 
organism has also been regarded as pathogenic in a number of reported instances. Meningitis (Aronson and Alderman, 1943 ; Rabinowitz and Schiffrin, 1952), bacterial endocarditis (Hawe and Hughes, 1954), infections of the urinary tract (Wheat et al., 1951 ; Taylor and Keane, 1962), empyema (Papapanagiotou and Aligizakis, 1959), and septicaemia (Graber et al., 1960); Emanuel et al., 1963) have all been reported as being due to $S$. marcescens infection. The case described by Emanuel et al. (1963) followed rupture of the dialysing coil during haemodialysis.

The presence of mucus at the site of infection has been postulated as a means of increasing the virulence of this organism (Taylor and Keane, 1962). Gale and Lord (1957) have shown experimentally that the usually low virulence of $S$. marcescens for animals is greatly enhanced by the presence of mucus in the inoculum. In a critical review of $S$. marcescens infections, Haiby et al. (1961) concluded that there were seven reported fatalities in which the organism had played an important part. Of the three cases described above one patient (Case 1) would seem to have died partly as a result of peritonitis, since the infection remained active until his death, prior to and after which $S$. marcescens was obtained in pure culture from the peritoneum.

The association of $S$. marcescens with other enteric organisms, particularly with the enterobacteria, is noteworthy (Wheat et al., 1951 ; Papapanagiotou and Aligizakis, 1959 ; Taylor and Keane, 1962 ; Emanuel et al., 1963). E. coli and Ps. aeruginosa, both of which were found in instances reported here, are the most commonly associated bacteria. This suggests that $S$. marcescens is more liable to show enhanced virulence not only in chronic debility (Haiby et al., 1961) but also where infection by another organism has been established.

Just as important is the significance of the organism as an indicator of cross-infection and flaws in technique, especially of catheterization. The failure to discover the source of the organism in these three cases is puzzling, but Taylor and Keane (1962), who described seven instances of cross-infection by $S$. marcescens following bladder catheterization, were also unable to trace the source of the organism.

There is no doubt, however, that $S$. marcescens can easily be missed in laboratory culture. This can be avoided by retaining all cultures for a further 48 hours at room temperature after initial incubation. Under these conditions the typical red pigment produced can be readily identified.

The treatment of $S$. marcescens infection with antibiotics, especially in the presence of renal failure, presents special prob- lems. This organism is commonly associated with known pathogens, such as Ps. aeruginosa and $E$. coli, which may have a limited and entirely different antibiotic sensitivity. Since the evidence in these and other cases suggests that the isolation of $S$. marcescens should not be lightly regarded, two appropriate antibiotics may be needed to combat that organism as well as any concomitant pathogen. With a few exceptions, all recently reported strains have shown marked resistance to most antibiotics with the exception of neomycin and kanamycin. It has been suggested that treatment with either of these antibiotics should be begun as soon as the organism is isolated, without waiting for the results of sensitivity tests (Emanuel et al., 1963). However, the systemic use of kanamycin in a patient with renal failure may be complicated by severe ototoxic effects arising from these antibiotics, so that strict control of therapy by frequent estimation of the antibiotic levels in the patient's serum is essential.

\section{Summary}

Three instances, two fatal, of acute renal failure are described, in which treatment by peritoneal dialysis was complicated by Serratia marcescens infection of the peritoneum. This organism seems to be pathogenic in a variety of sites and circumstances, particularly in association with chronic debility and with other established bacterial infections. The very limited antibiotic sensitivity of the organism is discussed.

We thank Wing Commander T. C. Nicol for access to the clinical notes, Air Commodore W. P. Stamm for his criticism and advice, and the Director-General of Medical Services, Royal Air Force, for permission to publish.

\section{REFERENCES}

Aronson, J. D., and Alderman, I. (1943). F. Bact., 46, 261. Burket, L. W., and Burn, C. G. (1937). $\dot{\mathfrak{f}}$. dent. Res., 16, 521. Emanuel, B., Goldin, M., and Brauer, M. (1963). F. Urol., 90, 34. Gale, D., and Lord, J. D. (1957). F. Amer. med. Ass., 164, 1328. Graber, C. D., Tumbusch, W. T., Rudnicki, R. P., and Vogel, E. H. (1960). Surg. Gynec. Obstet., 110, 443.

Haiby, G., McFarland, R. B., and Moore, G. H. (1961). Amer. J. clin. Path., 36, 256.

Hawe, A. J., and Hughes, M. H. (1954). Brit. med. f., 1, 968.

Klein, E. (1894). F. Path. Bact., 2, 217.

McEntegart, M. G., and Porterfield, J. S. (1949). Lancet, $2,596$.

Papapanagiotou, J., and Aligizakis, Ch. (1959). F. clin. Path., 12, 170.

Rabinowitz, K., and.Schiffin, R. (1952). Harefulah, 42, 90.

Taylor, G., and Keane, P. M. (1962). F. clin. Path., 15, 145.

Taylor, G., and Keane, P. M. (1962), F. clin. Path., 15, 145. Med., 88, 461.

Wilson, G. S., and Miles, A. A. (1955). Topley and Wilson's Principles of Bacteriology and Immunity, 4th ed., vol. 1, p. 731. Arnold. London.

\section{Preliminary Communications}

\section{Determination of the Emptying-time of the Stomach by Use of Enteric-coated Barium Granules}

Brit. med. F., 1965, 1, 1537-1539

An easy method of assessing the cmptying-time of the stomach has not yet been described. Methods which entail repeated intubation are of doubtful acceptability to the average patient.

Van Liere and Sleeth (1935, 1940) employed barium sulphate mixed with a standard carbohydrate meal and assessed the emptying-time by serial radiography. Although their technique gave constant results for individuals, it was criticized by W. M. Capper (personal communication, 1964) on the grounds that barium sulphate is an irritant to the stomach and promotes early emptying. It was also claimed that the heavy barium sulphate would separate and empty before the meal. A similar technique was employed by Oberhelman (1959), who mixed barium sulphate with a meal consisting of meat, eggs, milk, toast, and cereal. With this technique the emptying-time for normal subjects was from three to six hours; in patients with gastric ulcer it was 8 to 15 hours.

To avoid the criticisms which have been made in regard to barium sulphate we have used it in enteric-coated granules. This avoids any possiblity of irritation of the gastric mucosa by the barium sulphate and also gives a compound similar in specific gravity to food. 